\title{
EVENTOS EXTREMOS DE PRECIPITAÇÃO NO ESTADO DO CEARÁ E SUAS RELAÇÕES COM A TEMPERATURA DOS OCEANOS TROPICAIS
}

\author{
CARLOS ANTONIO COSTA DOS SANTOS ${ }^{1}$ E ANTONIO OCIMAR MANZI ${ }^{2}$
}

\author{
${ }^{1}$ Unidade Acadêmica de Ciências Atmosféricas, Universidade Federal de Campina Grande, (UFCG), \\ Campina Grande, PB, Brasil. \\ ${ }^{2}$ Instituto Nacional de Pesquisas da Amazônia (INPA), Manaus, AM, Brasil \\ carlostorm@gmail.com,manzi@inpa.gov.br.
}

Recebido Janeiro 2010 - Aceito Maio 2010

\begin{abstract}
RESUMO
O principal objetivo deste trabalho é prover informações sobre as tendências recentes dos eventos extremos de precipitação sobre o Estado do Ceará, associando esses eventos extremos às anomalias de Temperatura da Superfície do Mar (TSM) nos Oceanos Pacífico e Atlântico. Foram utilizados dados pluviométricos de 18 postos de 1971 a 2006 e o método de Mann-Kendall foi utilizado na obtenção das tendências. Os resultados mostram que existem características de aumento nas intensidades das secas e diminuição dos eventos de precipitação forte, e que o aumento nas anomalias de TSM no Pacífico e ao norte do equador, no Oceano Atlântico, acarreta em um aumento do número de dias consecutivos secos no norte do Ceará. Os índices extremos de precipitação mostraram correlações negativas com as anomalias de TSM nas regiões do Pacífico do Atlântico Tropical Norte e positivas com a região do Atlântico Tropical Sul. Entretanto, para a região sul do Ceará, o comportamento dos dias consecutivos chuvosos indica que o período chuvoso é governado por outros sistemas atmosféricos, necessitando assim, ser melhor estudado para o entendimento desse comportamento.
\end{abstract}

Palavras - Chaves: Mudanças Climáticas, Semi-árido, El Niño, anomalia

\begin{abstract}
EXTREME RAINFALL EVENTS IN CEARÁ STATE AND ITS RELATIONSHIP WITH TROPICAL OCEANS TEMPERATURE

The main objective of this study is to provide information about recent trends of extreme rainfall over Ceará State, associating these extreme events with Sea Surface Temperature (SST) anomalies in the Pacific and Atlantic Oceans. Mann-Kendall method is applied to rainfall data of 18 rain gauges from 1971 to 2006 to obtain the trends. The results show that the drought intensity is increasing and the heavy precipitation events are decreasing and that the increase in the Pacific and north of equator Atlantic Ocean SST anomalies lead to an increase in the number of consecutive dry days in northern Ceará. Rainfall extreme indices have shown negative correlations with SST anomalies on Pacific and Tropical North Atlantic regions and positive with Tropical South Atlantic region. However, in southern Ceará region, the consecutive wet day behavior indicates that the rainy season is governed by other atmospheric systems, which needs to be more studied for better understanding.
\end{abstract}

Keywords: Climate Change, Semi-arid, El Niño, anomaly 


\section{INTRODUÇÃO}

Eventos extremos de precipitação, que incluem chuvas extremas e longos períodos de dias consecutivos secos, são os fenômenos atmosféricos mais perturbadores (Zin et al., 2010). A região Nordeste do Brasil (NEB) é conhecida pelos seus longos períodos de estiagem (secas), entretanto, nos últimos dois anos (2008 e 2009) essa região tem apresentado destaque na mídia pelos vários eventos de chuvas extremas, que ocorreram, especialmente sobre o Estado do Ceará. Tais eventos trouxeram alagamentos, perdas agrícolas, material e humana entre outras.

As possíveis mudanças climáticas poderão acarretar significativos impactos nos setores natural, social e econômico, assim como, os extremos do clima associados à temperatura e precipitação poderão também afetar consumo de energia, conforto humano e turismo (Santos et al., 2009). Kostopoulo e Jones (2005) afirmam que o aumento nas perdas econômicas devido a extremos de tempo e, especialmente, o aumento na perda de vidas, tem sido regularmente notícias de jornais, despertando o interesse da comunidade científica em estudar os fenômenos climáticos.

O ciclo hidrológico e os recursos hídricos são afetados por mudanças no regime de precipitação. É possível que as mudanças no clima alterem a temperatura e a precipitação, e que aumentem a variabilidade dos eventos de precipitação, os quais poderão causar inundações e secas mais intensas e freqüentes (Dufek e Ambrizzi, 2008). Estudos têm mostrado que a freqüência e a persistência das secas deverá ser uma das consequiências do aquecimento global (Qian e Lin, 2005). No NEB as atividades agrícolas, quase que em sua totalidade, são baseadas na precipitação e o conhecimento de sua variabilidade é de extrema importância, principalmente para os setores sociais e econômicos.

O $4^{\circ}$ Relatório do IPCC (2007) mostra que nos últimos 100 anos vem acontecendo um progressivo aumento na concentração dos GEE (gases de efeito estufa) na atmosfera, sendo provocado, principalmente pelas atividades antrópicas. Segundo o IPCC (2007), para as próximas duas décadas, projeta-se um aquecimento de cerca de $0,2^{\circ} \mathrm{C}$ por década para uma faixa de cenários de emissões do RECE (Relatório Especial sobre Cenários de Emissões). Mesmo que as concentrações de todos os gases de efeito estufa e aerossóis se mantivessem constantes nos níveis do ano 2000, seria esperado um aquecimento adicional de aproximadamente $0,1^{\circ} \mathrm{C}$ por década. Até meados do século XXI, projeta-se que a vegetação semiárida tenderá a ser substituída por vegetação de terras áridas. Nas áreas mais secas, prevê-se que a mudança do clima acarrete a salinização e a desertificação das terras agrícolas.

Bernstein et al. (2007) indicaram que alguns eventos extremos de tempo têm mudado suas freqüências e/ou intensidade nos últimos 50 anos. Isto inclui um aumento na frequência de eventos de precipitação forte sobre muitas áreas do globo. Nesse período, a precipitação aumentou significativamente nas partes leste das Américas do Sul e Norte, norte da Europa e centro da Ásia, enquanto diminuiu no Sahel, Mediterrâneo, sul da África e partes do sul da Ásia.

Haylock et al. (2006), com o objetivo de estudar mudanças climáticas regionais, fizeram uma análise da precipitação sobre a América do Sul e observaram uma tendência de aumento do total anual de chuva sobre o NEB. O estudo realizado por Santos e Brito (2007), utilizando índices de extremos climáticos e correlacionando-os com as anomalias de temperatura da superfície do mar (TSM), também mostra tendência de aumento da precipitação total anual nos estados da Paraíba e Rio Grande do Norte. Santos et al. (2009) analisaram diferentes índices de deteç̧ão de mudanças climáticas, baseados em dados de precipitação diária (1935 - 2006) para o Estado do Ceará, e identificaram que ocorreram mudanças locais na precipitação e um visivel aumento nas condições de umidade sobre a região.

Xavier et al. (2003) e Santos e Brito (2007) mostraram que a precipitação sobre o NEB é bastante sensível a extremos de TSM no Oceano Pacífico Equatorial associado ao evento El Niño Oscilação Sul (ENOS) (Ropelewski e Halpert, 1987), assim como, as anomalias de TSM do Atlântico Tropical associado ao Dipolo do Atlântico. Vale salientar que os primeiros estudos mostrando a dependência da precipitação no NEB com as anomalias de TSM no Atlântico tropical, foram desenvolvidos por Hastenrath e Heller (1977) e Moura e Shukla (1981).

As variabilidades temporal e espacial da precipitação são determinantes para as condições climáticas do Semiárido brasileiro. Nesse contexto, o intuito deste trabalho é prover informações sobre as tendências recentes dos eventos extremos de precipitação sobre o Estado do Ceará, associando esses eventos extremos às anomalias de TSM nos Oceanos Pacífico Equatorial e do Atlântico tropical. Esse trabalho é de fundamental importância para o Estado do Ceará, uma vez que alterações no clima poderão influenciar o setor sócio-econômico, a população, assim como, causar sérios impactos ambientais.

\section{DADOS E METODOLOGIA}

Os dados de precipitação diária foram disponibilizados pela Fundação Cearense de Meteorologia (FUNCEME). Foram escolhidos 18 postos, que apresentaram dados sem falhas no período de 1971 a 2006, e representam todas as microrregiões do Estado (Figura 1). A consistência espacial e temporal dos valores diários da precipitação foi empregada para minimizar as influências associadas com a heterogeneidade temporal.

Os quatro índices de eventos climáticos extremos derivados da precipitação diária, utilizados nessa pesquisa, 
juntamente com suas definições conforme o Expert Team on Climate Change Detection Monitoring and Indices (ETCCDMI), estão mostrados na Tabela 1. Na avaliação da homogeneização dos dados e no cálculo dos índices foi utilizada a metodologia de Zhang et al. (2005) implementada no software RClimdex 1.9.0, desenvolvido e mantido pelos pesquisadores Xuebin Zhang e Feng Yang do Serviço de Meteorologia do Canadá. O propósito do controle de qualidade foi identificar erros nos dados, conforme proposto por Alexander et al. (2006), assim como, a aplicação da metodologia proposta por Viney e Bates (2004).

O RClimdex 1.9.0 fornece, para todos os índices, dados estatísticos, tais como: tendência linear calculada pelo método de mínimos quadrados; nível de significância estatística da tendência (valor p); coeficiente de determinação $\left(\mathrm{r}^{2}\right)$ e erro padrão de estimativa; assim como os gráficos das séries anuais. Como os dados não se ajustaram a uma distribuição de frequência normal, foi utilizado o teste não-paramétrico de
Mann-Kendall (Sneyers, 1990) para identificar se as tendências apresentam nível de significância estatística de $95 \%(\mathrm{p}<0,05)$, conforme mostrado na Tabela 2.

Conforme apresentado anteriormente, diversos trabalhos têm mostrado a relação de dependência entre o total anual de precipitação no NEB com as anomalias de TSM dos Oceanos Pacífico Equatorial e Atlântico Tropical. Santos e Brito (2007) mostraram que para a agricultura e armazenamento de águas nessa região, não apenas o total anual de precipitação, mas também os seus extremos e períodos consecutivos secos e chuvosos são importantes para o Semi-árido brasileiro. Logo, os índices de extremos climáticos foram correlacionados com as anomalias de TSM dos referidos oceanos. As anomalias de TSM foram obtidas das áreas conhecidas por Niño 1+2, Niño 3, Niño 3.4 e Niño 4 (Figura 2) no Oceano Pacífico Equatorial, e as regiões de TNAI e TSAI (Figura 3) no Oceano Atlântico Tropical, conforme descrito em Oliveira (1999) e no sítio eletrônico do Climate Prediction Center (http://www.cpc.noaa.

Tabela 1 - Índices climáticos extremos dependentes da precipitação pluvial diária, com suas definições e unidades. O PRCP é o valor da precipitação diária. $\mathrm{PRCP} \geq 1 \mathrm{~mm}$ representa um dia úmido e $\mathrm{PRCP}<1 \mathrm{~mm}$, um dia seco.

\begin{tabular}{|c|c|c|c|}
\hline ID & Nome do Indicador & Definição & Unidade \\
\hline DCS & Dias consecutivos secos & $\begin{array}{l}\text { Número máximo de dias consecutivos } \\
\text { com } \mathrm{PRCP}<1 \mathrm{~mm}\end{array}$ & dias \\
\hline DCU & Dias consecutivos úmidos & $\begin{array}{l}\text { Número máximo de dias consecutivos } \\
\text { com } P R C P \geq 1 \mathrm{~mm}\end{array}$ & dias \\
\hline R95p & Dias muito úmidos & $\begin{array}{l}\text { Precipitação anual total em que } \\
\text { PRCP }>95 \text { percentil }\end{array}$ & $\mathrm{mm}$ \\
\hline Rx5day & $\begin{array}{l}\text { Quantidade máxima de precipitação } \\
\text { em cinco dias consecutivos }\end{array}$ & $\begin{array}{l}\text { Máximo anual de precipitação em } 5 \text { dias } \\
\text { consecutivos }\end{array}$ & $\mathrm{mm}$ \\
\hline
\end{tabular}

\begin{tabular}{|c|}
\hline Localidades \\
\hline 1 - Crato $(-7.23 /-39.40)$ \\
\hline 2 - Fortaleza $(-3.73 /-38.57)$ \\
\hline 3 - Iguatu $(-6.37 /-39.30)$ \\
\hline 4 - Limoeiro do Norte $(-5.13 /-38.10)$ \\
\hline $5-$ Sobral $(-3.70 /-40.35)$ \\
\hline 6 - Tauá $(-6.02 /-40.28)$ \\
\hline 7 - Independência $(-5.45 /-40.48)$ \\
\hline 8 - Assaré (-6.87/-39.87) \\
\hline 9 - Nova Russas $(-4.73 /-40.57)$ \\
\hline 10 - Canindé $(-4.35 /-39.30)$ \\
\hline 11 - Iracema $(-5.82 /-38.30)$ \\
\hline 12 - Quixeramobim (-5.20/-39.28) \\
\hline 13 - Aracatí (-4.57/-37.77) \\
\hline $14-$ Brejo Santo (-7.50/-38.98) \\
\hline 15 - Baturité (-4.33/-38.87) \\
\hline 16 - Itapipoca $(-3.50 /-39.57)$ \\
\hline $17-$ Granja $(-3.13 /-40.83)$ \\
\hline 18 - Viçosa do Ceará $(-3.57 /-41.08)$ \\
\hline
\end{tabular}

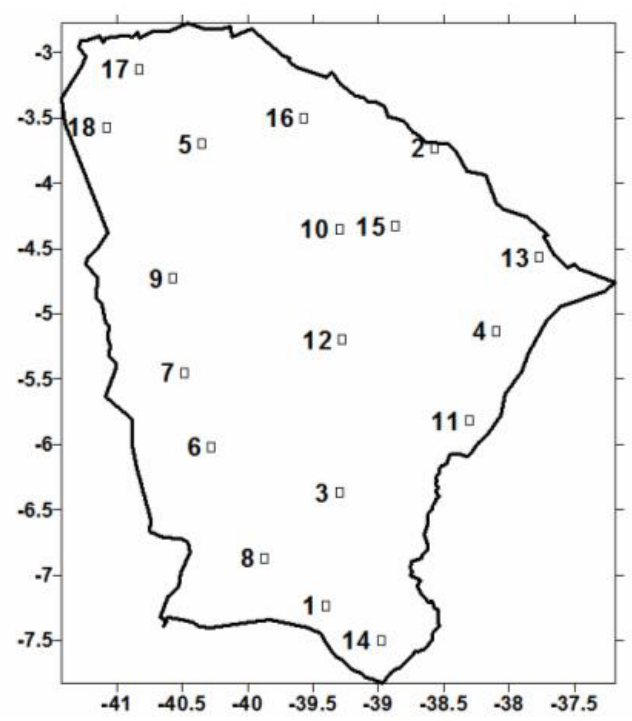

Figura 1 - Distribuição espacial das 18 localidades escolhidas no Estado do Ceará, com os seus respectivos números identificadores e coordenadas geográficas. (Fonte: Santos et al., 2009) 
gov/products/analysis_monitoring/ensostuff/nino_regions. shtml) da National Oceanic and Atmospheric Administration (NOAA).

Com o intuito de analisar o comportamento dos índices de extremos climáticos para diferentes partes do Ceará, o Estado foi dividido nas porções norte e sul, ambas contendo nove estações meteorológicas. Foi utilizado o método de Pearson na obtenção da correlação entre as anomalias de TSM, no período de 1971 a 2006, e os índices de extremos climáticos obtidos a partir da média aritmética anual das nove estações. Os índices foram correlacionados individualmente com a anomalia de TSM de cada área estudada e as correlações, com significância estatística ao nível de 95\% ( $<<0.05)$, estão apresentadas nas Tabelas 3 e 4.

\section{RESULTADOS}

Na Tabela 2 estão apresentados os valores das tendências dos índices climáticos extremos, obtidas pelo método de MannKendall e com nível de significância de $95 \%(\mathrm{p}<0,05)$ para os valores em destaque e negrito e de $90 \%(\mathrm{p}<0,1)$ apenas para os valores em destaque, correspondentes as 18 localidades estudadas. Serão discutidas nessa pesquisa apenas as localidades que apresentaram tendências estatisticamente significativas.
Foram observados índices com alta significância estatística $(\mathrm{p}<0,05)$ apenas para a parte norte do Estado, sendo observados também, um aumento no número de dias consecutivos secos (DCS) para as estações de Canindé (Figura 4d) e Fortaleza (Figura 4e) com tendências de 0,20 dias/ano e de 0,27 dias/ ano, respectivamente. Estes resultados indicam que, se conservando a linearidade da tendência, em 10 anos o número de dias consecutivos secos terão aumentado 2 e 3 dias para as respectivas localidades.

Os dias consecutivos úmidos (DCU) mostraram resultados opostos entre as localidades, ou seja, Baturité (Figura 4c) com tendência positiva e indicando um aumento

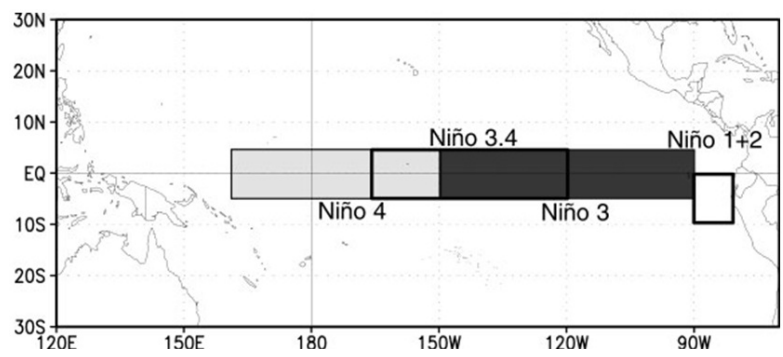

Figura 2 - Regiões do Oceano Pacífico Equatorial, Niño 1+2, Niño 3, Niño 3.4 e Niño 4. (Fonte: NOAA, 2010)

Tabela 2 - Tendências dos índices climáticos extremos dependentes da precipitação pluvial diária para 18 localidades distribuídas no Estado do Ceará (norte e sul). Os valores realçados em negrito apresentaram alta significância estatística $(\mathrm{p}<0,05)$, os outros valores realçados apresentaram boa significância estatística $0,05<\mathrm{p}<0,1$.

\begin{tabular}{|c|c|c|c|c|c|c|c|}
\hline Norte - CE & Latitude & Longitude & $\begin{array}{c}\text { Altitude } \\
\text { (m) }\end{array}$ & $\begin{array}{c}\text { DCS } \\
\text { (dias/ano) }\end{array}$ & $\begin{array}{c}\mathrm{DCU} \\
\text { (dias/ano) }\end{array}$ & $\begin{array}{c}\text { R95p } \\
\text { (mm/ano) }\end{array}$ & $\begin{array}{l}\text { Rx5day } \\
\text { (mm/ano) }\end{array}$ \\
\hline Aracatí & $-4,57$ & $-37,77$ & 5 & 0,13 & 0,18 & $-0,23$ & $-0,26$ \\
\hline Baturité & $-4,33$ & $-38,87$ & 175 & 0,08 & 0,31 & 0,02 & 0,04 \\
\hline Canindé & $-4,35$ & $-39,3$ & 148 & 0,2 & 0,07 & 0,06 & $-0,01$ \\
\hline Fortaleza & $-3,73$ & $-38,57$ & 21 & 0,27 & $-0,28$ & $-0,07$ & 0,03 \\
\hline Granja & $-3,13$ & $-40,83$ & 10 & $-0,07$ & $-0,08$ & $-0,14$ & $-0,09$ \\
\hline Itapipoca & $-3,5$ & $-39,57$ & 108 & $-0,18$ & $-0,28$ & 0,1 & 0,18 \\
\hline Nova Russas & $-4,73$ & $-40,57$ & 240 & 0,18 & $-0,26$ & $-0,06$ & 0,02 \\
\hline Sobral & $-3,7$ & $-40,35$ & 69 & 0,03 & $-0,05$ & 0,11 & 0,13 \\
\hline Viçosa do Ceará & $-3,57$ & $-41,08$ & 740 & 0,05 & 0,12 & $-0,23$ & $-0,01$ \\
\hline \multicolumn{8}{|l|}{ Sul - CE } \\
\hline Assaré & $-6,87$ & $-39,87$ & 442 & 0,16 & 0,03 & $-0,08$ & $-0,09$ \\
\hline Brejo Santo & $-7,5$ & $-38,98$ & 381 & 0,13 & $-0,1$ & $-0,14$ & $-0,13$ \\
\hline Crato & $-7,23$ & $-39,4$ & 426 & 0.01 & 0.03 & -0.97 & -1.30 \\
\hline Iguatu & $-6,37$ & $-39,3$ & 217 & 0,02 & 0,01 & $-0,08$ & $-0,03$ \\
\hline Independência & $-5,45$ & $-40,48$ & 343 & 0,08 & 0,08 & 0,11 & 0,03 \\
\hline Iracema & $-5,82$ & $-38,3$ & 131 & 0,03 & 0,07 & 0 & $-0,01$ \\
\hline Limoeiro do Norte & $-5,13$ & $-38,1$ & 30 & 0,12 & 0,02 & $-0,2$ & $-0,21$ \\
\hline Quixeramobim & $-5,2$ & $-39,28$ & 191 & 0,09 & 0,06 & $-0,16$ & $-0,2$ \\
\hline Tauá & $-6,02$ & $-40,28$ & 402 & 0,1 & 0,09 & 0,12 & 0,16 \\
\hline
\end{tabular}


de aproximadamente 3 dias/década, enquanto as localidades de Itapipoca (Figura 4f) e Nova Russas (Figura 4g) apresentaram tendências negativas da ordem de 3 dias/década. Esses resultados mostram a alta variabilidade espacial dos dias consecutivos úmidos na região. O índice R95p, que representa os dias muito úmidos, apresentou tendência predominante de diminuição, o que pode ser evidenciado nas estações de Aracatí

Tabela 3 - Correlações entre as médias anuais dos índices extremos de precipitação e as anomalias anuais de temperatura da superfície do mar nas regiões dos Oceanos Atlântico e Pacífico para a parte norte do Ceará, com significância estatística de $95 \%$.

\begin{tabular}{lcccc}
\hline & DCS & DCU & R95p & Rx5day \\
\hline Niño 1+2 & 0.290 & - & - & - \\
Niño 3 & 0.191 & - & - & - \\
Niño 3.4 & 0.159 & - & -0.323 & -0.328 \\
Niño 4 & 0.127 & - & -0.184 & -0.159 \\
TNAI & 0.270 & -0.222 & - & -0.147 \\
TSAI & 0.103 & 0.175 & 0.223 & 0.170 \\
\hline
\end{tabular}

Tabela 4 -Correlações entre as médias anuais dos índices extremos de precipitação e as anomalias anuais de temperatura da superfície do mar nas regiões dos Oceanos Atlântico e Pacífico para a parte sul do Ceará, com significância estatística de $95 \%$.

\begin{tabular}{lcccc}
\hline & DCS & DCU & R95p & Rx5day \\
\hline Niño 1+2 & 0.105 & -0.326 & - & -0.229 \\
Niño 3 & 0.114 & -0.253 & - & -0.150 \\
Niño 3.4 & 0.147 & -0.157 & -0.263 & -0.028 \\
Niño 4 & 0.190 & -0.040 & -0.203 & -0.059 \\
TNAI & 0.121 & 0.114 & -0.222 & -0.077 \\
TSAI & -0.073 & -0.012 & 0.115 & 0.094 \\
\hline
\end{tabular}

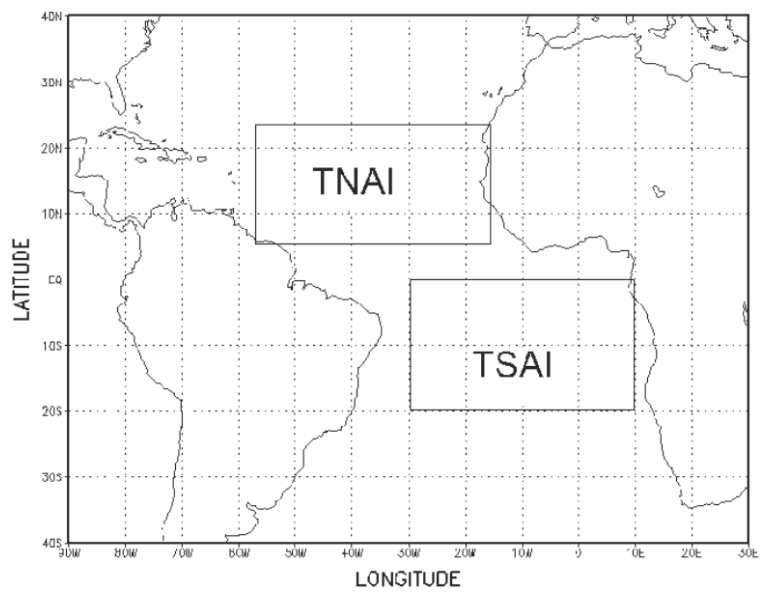

Figura 3 - Regiões do Oceano Atlântico Tropical usadas para o cálculo dos índices TNAI e TSAI, associados ao padrão de dipolo de anomalias das temperaturas do Oceano Atlântico. (Fonte: Menezes et al., 2008)
(Figura 4a) e Viçosa do Ceará (Figura 4h) com tendências de -2,3 $\mathrm{mm} /$ década para ambas as estações. $O$ total de precipitação em 5 dias consecutivos ( $\mathrm{Rx} 5$ day) mostrou tendência de diminuição (-2,6 mm/década) para o município de Aracatí (Figura 4b). Os resultados apontam para aumento nas intensidades das secas e diminuição dos eventos forte de precipitação, assim como, indicam a alta variabilidade do período chuvoso com valores positivos e negativos para os dias consecutivos úmidos.

Conforme descrito anteriormente, na parte sul do Ceará os índices DCS e DCU não apresentaram tendências com significância estatística. $\mathrm{O}$ índice $\mathrm{R} 95 \mathrm{p}$ apresentou tendência de diminuição $(-0,20 \mathrm{~mm} / \mathrm{ano})$, com $\mathrm{p}<0,1$, para Limoeiro do Norte (Figura 5b). Da mesma forma, o índice Rx5day evidenciou diminuição para as estações de Crato (Figura 5a), Limoeiro do Norte (Figura 5c) e Quixeramobim (Figura 5d) mostrando tendências anuais de $-1,3 \mathrm{~mm},-0,21 \mathrm{~mm}$ e $-0,20 \mathrm{~mm}$, respectivamente. As tendências apontam para diminuição dos eventos extremos de precipitação na parte sul do Ceará. Santos et al. (2009) analisaram as mesmas localidades estudadas e a base de dados, entretanto, utilizaram o período de dados de 1935 a 2006 e encontraram um aumento nas condições de umidade sobre a parte sul do Estado. Esses resultados indicam que a tendência de aumento da série de 1935 a 1970, foi predominante sobre a tendência de diminuição apresentada pela série de 1971 a 2006, visto que foi utilizada a mesma base de dados. Essa predominância de aumento na tendência foi determinante para o comportamento dos resultados encontrados por Santos et al. (2009).

Estudos como os de Qian e Lin (2005) têm mostrado que as maiores alterações nas tendências dos extremos climáticos têm ocorrido nas últimas três décadas com a intensificação das emissões de gases de efeito estufa, mau uso do solo entre outros. Baseado nessas informações, o presente trabalho foi desenvolvido com a preocupação de analisar se as tendências dos índices de extremos de precipitação, apresentados por Santos et al. (2009), se mantêm quando utilizado somente o período de 1971 a 2006. Os resultados apresentados na Tabela 2 mostram tendências diferentes dos apresentados por Santos et al. (2009), pois estações como as de Canindé e Fortaleza não mostraram tendências com significância estatística para o índice DCS no período de 1935 a 2007. Entretanto, foi observado quando analisado somente o período mais recente (1971 a 2006). O contrário também foi evidenciado, ou seja, estações que apresentavam tendências significativas, não foram observadas quando utilizados os dados de 1971 a 2006. Com relação ao índice DCU, a estação de Nova Russas concordou com os resultados mostrados por Santos et al. (2009). Os índices de precipitação extrema (R95p e Rx5day) obtidos nesse estudo apresentam tendências diferentes daquelas apresentadas no trabalho de Santos et al. (2009), pois apresentaram tendências 
negativas para todas as localidades com significância estatística, o oposto aos apresentados no referido trabalho, que identificaram aumento das condições de umidade no Ceará. Isto ocorre devido ao fato dos estudos utilizarem a mesma base de dados, porém, períodos diferentes.

Conforme mencionado anteriormente, o clima do NEB tem sua variabilidade associada a padrões de anomalias da TSM
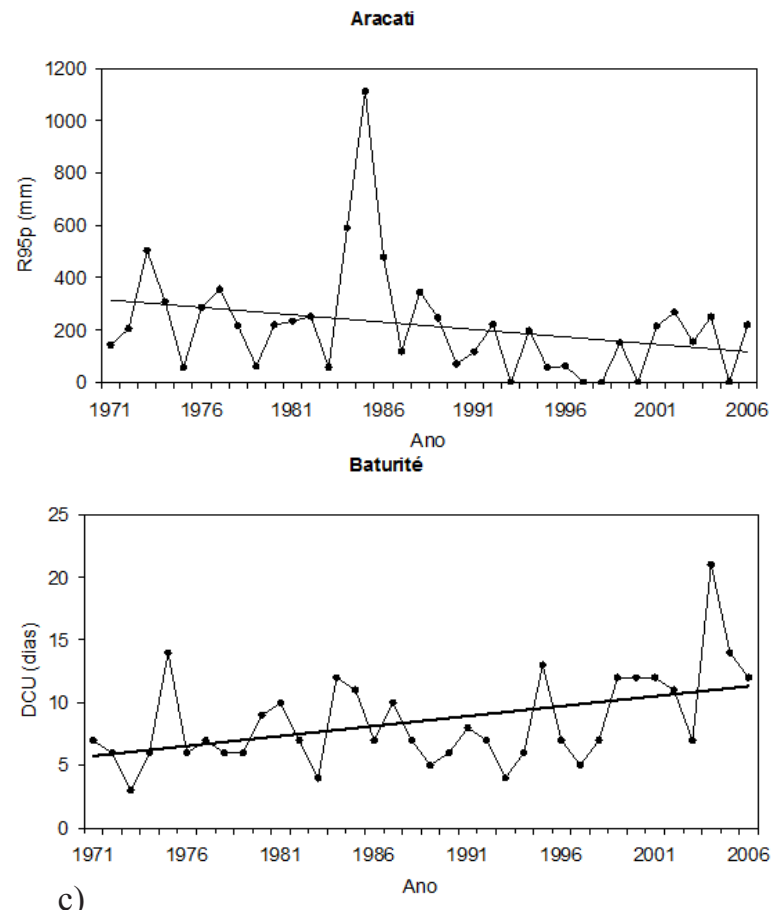

c)

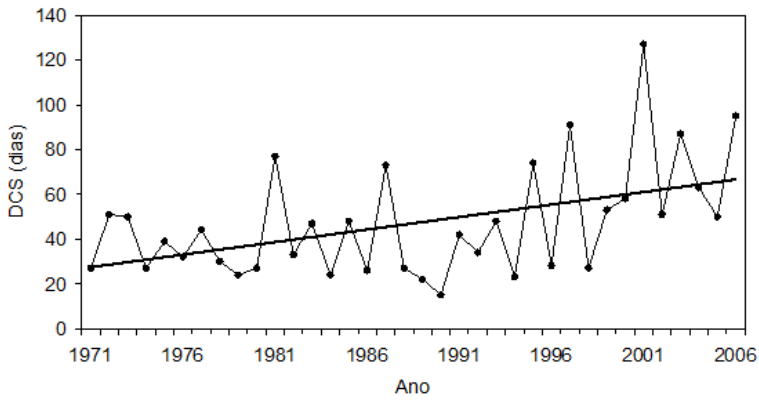

e)

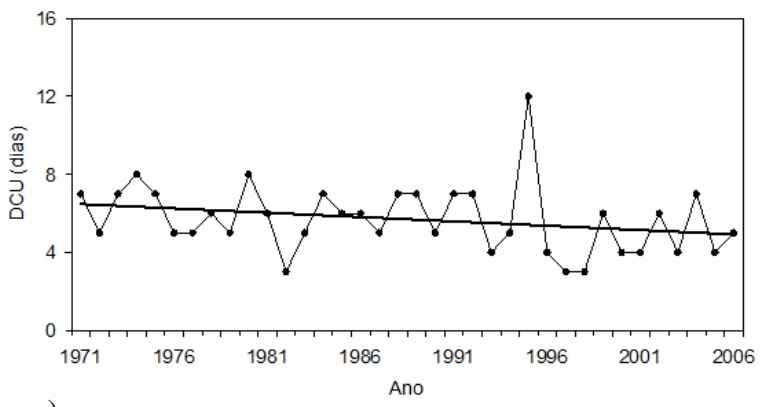

g) dos Oceanos Pacífico Equatorial (El Niño/La Nina) e Atlântico Tropical (Dipolo meridional de anomalias de TSM). O ENOS e o Dipolo do Atlântico afetam diretamente o posicionamento da Zona de Convergência Intertropical (ZCIT), que por sua vez, influencia a distribuição de chuvas sobre o NEB. Na Tabela 3 estão mostrados os valores das correlações, com significância estatística de $95 \%(\mathrm{p}<0,05)$, entre as médias anuais dos índices
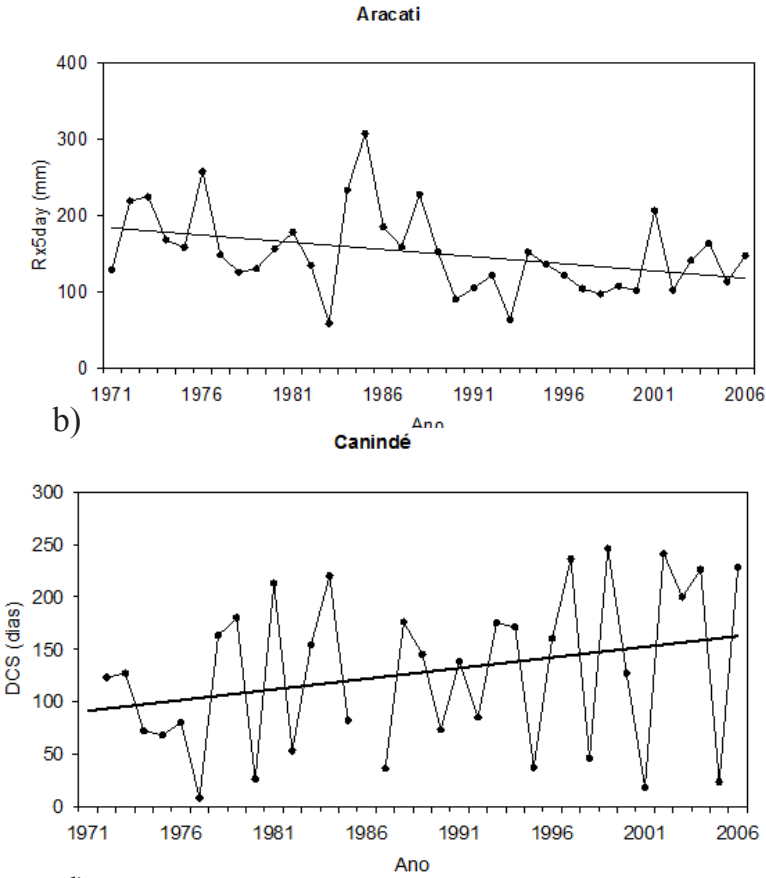

d)

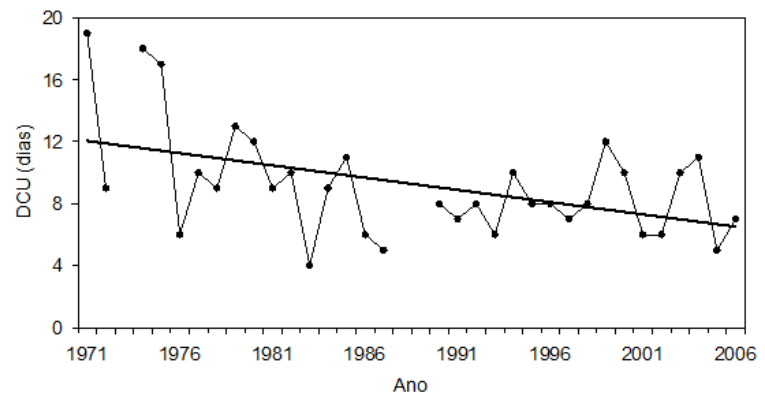

f)

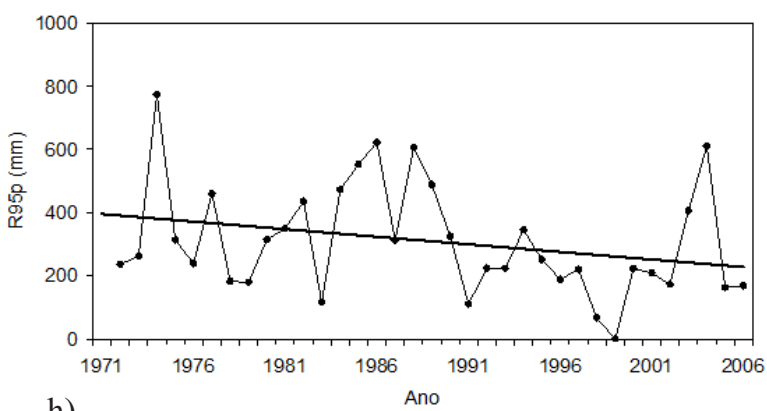

Figura 4 - Série temporal dos índices de extremos climáticos, dependentes da precipitação pluvial diária, com significância estatística para parte norte do Estado do Ceará 
extremos de precipitação e as anomalias anuais de TSM nas regiões escolhidas dos Oceanos Pacífico Equatorial e Atlântico Tropical para a região norte do Ceará. A tabela mostra que todas as regiões de Niño (Pacífico Equatorial) e de TNAI/TSAI (Atlântico Tropical) apresentam correlações positivas com o índice DCS, caracterizando que aumento nas temperaturas da superfície desses oceanos acarreta em um aumento do número de dias consecutivos secos no norte do Ceará. O índice DCU, que corresponde ao número de dias consecutivos chuvosos, apresentou correlações negativas com a região de TNAI e positiva com a de TSAI. Estes resultados evidenciam que a anomalia positiva de TSM ao norte do equador, no Oceano Atlântico, induz que a ZCIT se desloque para o norte, inibindo os eventos chuvosos, e aumentando o número de dias consecutivos secos sobre o norte do Ceará. Entretanto, anomalias positivas de TSM ao sul do equador, no Oceano Atlântico, induz uma situação contrária à descrita anteriormente. Os índices extremos de intensidade da precipitação (R95p e Rx5day) mostraram correlações negativas com as anomalias de TSM nas regiões de Niño e de TNAI e positivas com a região de TSAI. Conforme já discutido, anomalias positivas de TSM nas regiões de Niño e de TNAI inibem a formação de chuvas sobre o norte do NEB. No entanto, anomalia positiva de TSM, na região de TSAI, favorece a formação de chuvas nessa região. Santos e Brito (2007) encontraram resultados semelhantes para os Estados da Paraíba e Rio Grande do Norte. Os resultados concordam com

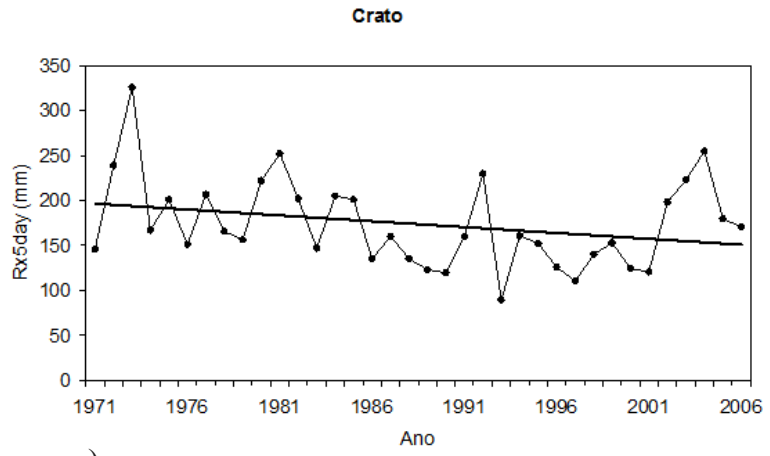

a)

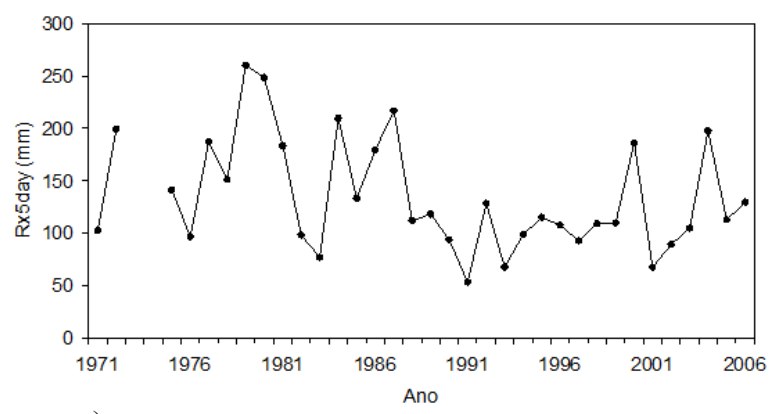

c)
Hastenrath e Heller (1977), Moura e Shukla (1981), Uvo et al. (1998) e Kucharski et al. (2008).

Na Tabela 4 estão mostrados os valores das correlações entre as médias anuais dos índices extremos de precipitação e as anomalias anuais de TSM para a região sul do Ceará. Os resultados são bastante semelhantes aos apresentados para a região norte do Estado, além de mostrar maior número de correlações com significância estatística. Entretanto, os índices DCS e DCU apresentam comportamentos distintos daqueles apresentados para o norte do Estado. A correlação entre o DCS e a região de TNAI é negativa, reforçando o fato de que com anomalia positiva de TSM no sul do equador a ZCIT se desloca para o sul favorecendo a formação de chuvas sobre o NEB e, com isso, a diminuição do número de dias consecutivos secos concordando com Hastenrath e Heller (1977), Moura e Shukla (1981), Uvo et al. (1998), Santos e Brito (2007) e Kucharski et al. (2008). Vale salientar, que o índice DCU apresentou um comportamento inesperado quando correlacionado com as regiões do Atlântico Tropical, pois mostrou correlação positiva com o TNAI e negativa com o TSAI, quando deveria ser o contrário. Isso indica que os comportamentos dos dias consecutivos chuvosos, nessa parte do Ceará, são governados por outros sistemas atmosféricos, tais como: bandas de nebulosidades associada às Frentes Frias, Distúrbios de Leste, Ciclones na média e alta troposfera do tipo baixa fria conhecidos como Vórtice Ciclônico de Ar Superior (VCAS), as Brisas terrestre e marítima e um mecanismo de

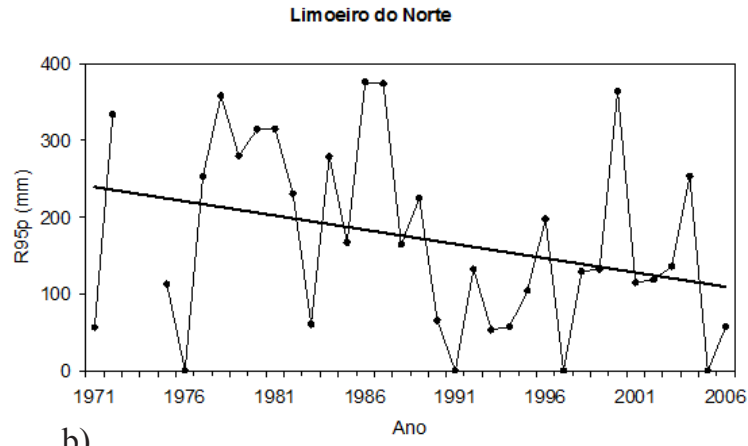

b)

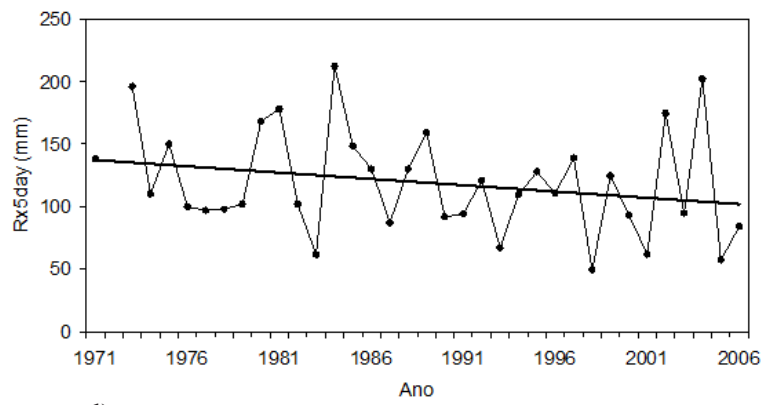

d)

Figura 5 - Série temporal dos índices de extremos climáticos, dependentes da precipitação pluvial diária, com significância estatística para parte sul do Estado do Ceará 
escala planetária conhecido como a Oscilação 30-60 dias (Alves et al., 2006). Alves et al. (2006) realizaram um diagnóstico dos principais mecanismos atmosféricos associados à ocorrência de precipitação intensa sobre a região Nordeste do Brasil (NEB) no mês de janeiro de 2004 (que foi um dos mais intensos no registro histórico dos últimos 40 anos), e observaram a influência dos mecanismos citados acima na precipitação do NEB. Contudo, os resultados encontrados nesse estudo necessitam ser melhor estudados para o entendimento desse comportamento dos dias consecutivos chuvosos.

\section{CONCLUSÕES}

Os resultados mostram que apenas a região norte do Ceará apresenta tendências com alta significância estatística para os índices de eventos extremos. Existem características de aumento nas intensidades das secas e diminuição dos eventos de precipitação forte. Através da análise dos dias consecutivos úmidos é possível identificar a alta variabilidade espacial das tendências, apresentando resultados opostos entre as localidades. É possível observar que, quando analisado apenas o período com dados das últimas três décadas, os resultados foram significativamente diferentes dos apresentados por Santos et al. (2009), indicando que existem comportamentos diferentes entre os períodos de 1935 a 1970 e 1971 a 2006 que precisam ser estudados, pois foi utilizada a mesma base de dados, porém com períodos diferentes.

Aumento nas anomalias de temperaturas da superfície dos Oceanos Pacífico Equatorial e Atlântico Tropical, acarreta em um aumento do número de dias consecutivos secos no norte do Ceará, e a anomalia positiva de TSM ao norte do equador, no Oceano Atlântico, induz que a ZCIT se desloque para o norte, inibindo os eventos chuvosos, e aumentando o número de dias consecutivos secos sobre o norte do Ceará. Os índices extremos de intensidade da precipitação (R95p e Rx5day) mostraram correlações negativas com as anomalias de TSM nas regiões de Niño e de TNAI e positivas com a região de TSAI, concordando com estudos já desenvolvidos para a região estudada.

Os índices extremos de precipitação, para a região sul do Ceará, apresentam características semelhantes às apresentadas para a região norte do Estado. Entretanto, o comportamento dos dias consecutivos chuvosos, nessa parte do Ceará, indica que o período chuvoso é governado por outros sistemas atmosféricos, necessitando de se aprofundar o estudo desse comportamento, para um melhor entendimento dos fatores que afetam a precipitação sobre a região.

\section{AGRADECIMENTOS}

Os autores agradecem ao CNPq (Conselho Nacional de Desenvolvimento Científico e Tecnológico) pela concessão de bolsa de pesquisa ao primeiro autor; a FUNCEME (Fundação Cearense de Meteorologia), por fornecer os dados de precipitação e aos pesquisadores Xuebin Zhang e Feng Yang do Serviço de Meteorologia do Canadá, por disponibilizarem gratuitamente o software RClimdex 1.9.0.

\section{REFERÊNCIAS BIBLIOGRÁFICAS}

ALEXANDER, L. V., et al. Global observed changes in daily climate extremes of temperature and precipitation. Journal of Geophysical Resources, v. 111, D05109, 2006.

ALVES, J. M. B., FERREIRA, F. F., CAMPOS, J. N. B., FILHO, F. A. S., SOUZA, E. B., DURAN, B. J., SERVAIN, J., STUDART, T. M. C. Mecanismos atmosféricos associados à ocorrência de precipitação intensa sobre o Nordeste do Brasil durante janeiro/2004. Revista Brasileira de Meteorologia, v. 21, p. 56-76, 2006.

BERNSTEIN, L., et al. Climate change 2007: synthesis report (IPCC 4th Assessment Report), 2007.

DUFEK, A. S., AMBRIZZI, T. Precipitation variability in São Paulo State, Brazil. Theoretical and Applied Climatology, v. 93, p. 167-178, 2008.

HASTENRATH, S., HELLER, L. Dynamics of climatic hazards in northeast Brazil. Quarterly Journal of the Royal Meteorological Society, v.103, p.77-92. 1977.

HAYLOCK, M. R., et al. Trends in total and extreme South American rainfall 1960-2000 and links with sea surface temperature. Journal of Climate, v. 19, p. 1490-1512, 2006.

INTERGOVERNMENTAL PANEL ON CLIMATE CHANGE (IPCC). Climate Change 2007 - The Physical Science Basis. Contribution of Working Group I to the Fourth Assessment Report of the IPCC. Cambridge Univ. Press, Cambridge, 2007.

KOSTOPOULO, E., JONES, P. D. Assesment of climate extremes in the Eastern Mediterrenean. Meteorology and Atmospheric Physics, v. 89, p. 69-85, 2005.

KUCHARSKI, F., POLZIN, D., HASTENRATH, S. Teleconnection mechanisms of Northeast Brazil droughts: modeling and empirical evidence. Revista Brasileira de Meteorologia, v. 23, p. 115-125, 2008.

MOURA, A. D., SHUKLA, J. On the dynamics of droughts in northeats Brazil: Observation, theory, and numerical experiments with a general circulation model. Journal of Atmospheric Sciences, v. 38, p.2653-2675, 1981.

OLIVEIRA, G. S. O EI Niño e Você - O fenômeno climático. São Paulo - SP, Transtec Editorial, 116 p., 1999.

QIAN, W., LIN, X. Regional trends in recent precipitation indices in China. Meteorology and Atmospheric Physics, v. 90, p. 193-207, 2005.

ROPELEWSKI, C. F., HALPERT, M. S. Global and Regional 
scale precipitation patterns associated with El Niño/Southern Oscillation. Monthly Weather Review, v. 115, 1606-1626, 1987.

SANTOS, C. A. C., BRITO, J. I. B. Análise dos índices de extremos para o semi-árido do Brasil e suas relações com TSM e IVDN. Revista Brasileira de Meteorologia, v. 22, n. 3, p. 303-312, 2007.

SANTOS, C. A. C., BRITO, J. I. B., RAMANA RAO, T. V., MENEZES, H. E. A. Tendências dos índices de precipitação no Estado do Ceará. Revista Brasileira de Meteorologia, v. 24, p. 39-47, 2009.

SNEYERS R. On the Statistical Analysis of Series of Observations. Technical Note No. 143, WMO No. 415 World Meteorological Organization: Geneva. 1990.

UVO, C. B., REPELLI, C. A., ZEBIAK, S. E., KUSHNIR, Y. The Relationships between Tropical Pacific and Atlantic SST and Northeast Brazil Monthly Precipitation. Journal of Climate, v. 11, p. 551-562, 1998.
VINEY, N. R., BATES, B. C. It never rains on Sunday: The prevalence and implications of untagged multi-day rainfall accumulations in the Australian high quality data set. International Journal of Climatology, v. 24, p. 11711192, 2004.

XAVIER, T. M. B. S., XAVIER, A. F. S., DIAS, M A F S., DIAS, P. L. S. Interrelações entre eventos ENOS (ENSO), a ZCIT (ITCZ) no Atlântico e a chuva nas bacias hidrográficas do Ceará. Revista Brasileira de Recursos Hídricos, v. 8, p. 111-126, 2003.

ZHANG, X., HEGERL, G., ZWIERS, F., KENYON, J. Avoiding inhomogeneity in percentile-based indices of temperature extremes. Journal of Climate, v. 18, p. 1641-1651, 2005. ZIN, W. Z. W., JAMALUDIN, S., DENI, S. M., JEMAIN, A. A. Recent changes in extreme rainfall events in Peninsular Malaysia: 1971 - 2005. Theoretical and Applied Climatology, v. 99, p. 303-314, 2010. 